ORIGINAL ARTICLE

\title{
EFFECTS OF CORE STABILITY EXERCISES VERSUS MYOFASCIAL RELEASE TECHNIQUE COMBINED WITH CORE STABILITY EXERCISES IN THE MANAGEMENT OF LOW BACK PAIN
}

\section{ABSTRACT \\ OBJECTIVES}

To compare the effectiveness of Core Stability with Myofascial release technique and Core Stability Exercises alone in the management of low back pain.

\section{STUDY DESIGN}

Randomized Control Trial (RCT).

\section{STUDY SETTINGS \& PARTICIPANTS}

The study was conducted on 44 patients with low back pain in the Department of Rehabilitation Sciences, at tertiary care Hospital.

\section{INTERVENTIONS}

The participants were divided into group $A(n=22)$ and group $B$ $(n=22)$ into Core Stability Exercises and Core Stability Exercises applied with Myofascial release technique respectively. Each group was provided interventions for 03 times weekly for 06 weeks, with a minimum of a 1 day gap between the 2 sessions. The Numeric Pain Rating Scale (NPRS) was utilized to assess pain, The Oswestry Disability Index (ODI) was utilized to assess the disability associated with LBP, and Rehabilitative Ultrasound imaging was used to provide multifidus muscle thickness.

\section{RESULTS}

The main effects analysis showed that the Core Stability Exercises applied with Myofascial release technique proved to be more effective than the Core Stability Exercises alone, reducing pain, functional disability and improving muscle mass of Lumber Multifidus $(p<0.05)$ in patients with low back pain.

\section{CONCLUSIONS}

Core Stability Exercises applied with Myofascial release techniques effectively reduce pain and functional disability when compared to core stability exercises.

\section{KEY WORDS}

Low Back Pain, Fascia, Exercise, Mobilization, Pain, Rehabilitation.

\section{Ahad Hasan}

Assistant Professor

Ziauddin College of Rehabilitation

Sciences

Ziauddin University

ahadhasan6691@gmail.com

\section{Syed Abid Mehdi Kazmi}

Associate Professor

Dr. Ziauddin Hospital syedabidmehdi@gmail.com

[Hasan A ,Kazmi SAM Effects of Core Stability Exercises VS MFR Technique Combined With Core Stability Exercises in the Management of LBP.Pak.j.rehabil.2020;9(2):24-29] DOI:10.36283/pjr.zU.9.2/006 


\section{INTRODUCTION}

Low back pain (LBP) is a leading cause of disability that bears the greatest burden, affecting 540 million people worldwide. LBP is a well-documented and an extremely common health problem that hinders the activities of daily living, compromises the quality of life'. According to World Health Organization Global Burden of Disease Study by World Health Organization (WHO-GBD) in 2010, "The most common reason of years lived with disability (YLDS) in the world was lower back-pain, followed by neck pain and other musculoskeletal disorders"2. In primary health care setting 60 to 80 percent of the population is predisposed to LBP sooner or later in their lifetime ${ }^{3}$. Gross Domestic Product (GDP) in most of the countries has been assessed to rise as high as 1.7 percent, due to the subsequent financial consequence of days lost from work, disability advantages related to occupation and health asset use which are caused by low back pain ${ }^{4}$. As reported by WHO's latest global burden of diseases study conducted in 2013, prevalence of low back pain has increased to $12.2 \%$, compared to the 2010 study conducted in 54 developing and developed countries, which was $11.9 \%$. LBP influences $40-60$ percent of working adults in western setting $s^{5}$. Kashif $M$, et al., conduct a study on Prevalence of LBP in Pakistan and demonstrated a prevalence of $6.5 \%$ to $44.1 \%^{6}$. Discussing about low back pain in detail, it is an indication of a pathological condition, that is neither a disease nor a diagnostic entity but have numerous concealed causes. LBP refers pain of variable duration and intensity within an area caused by a paradigm shift due to responses of external and internal stimuli. The term "low back pain" refers to discomfort in the back, arises from the lowest rib down to the gluteal region and extends into the legs 7 . However, the pain can also occur due to the underlying abdominal or pelvic pathology, irrespective of the spinal column. LBP is further categorized into acute and chronic back pain. Acute back pain is sudden intense pain and discomfort in the back, either felt for the first time in a patient's life or felt after six months of pain free interval ${ }^{8}$. On the contrary, chronic back pain is more severe, as it exists past 12 weeks in a patient's life. The causes of back pain, according to a classification in German National Disease management guide, are either known to be specific or non-specific'. Non-specific back pain, by definition, is the pain, which cannot be allocated to a known, specified pathology. The back pain is termed as non-specific when there are no correlations between the indications, physical and imaging findings, as it does not have a known pathoanatomical cause ${ }^{8}$. Reduced spinal movement whether it is general or segmental, will lead to pain and stiffness in lower back, followed by fear of spinal movement, inactivity or either disability ${ }^{10}$. Number of distinctive approaches for the management of low back pain consists of electrotherapy, spinal mobilization, and soft tissue techniques, followed by range of motion exercises ${ }^{11}$. The factors affecting the normal functioning of back are much diversified i.e. many of the disturbances are not related to any pathology, but may be rather caused by functional disturbances that are recognized by physical examination and can't be identified by imaging studies ${ }^{12}$, these include segmental dysfunction, altered spinal patterns of movement, muscle dysfunction, connective-tissue changes and systemic conditions $^{13}$, the weak trunk and abdominal musculature $^{14}$, leading to the lacking motor control of the spine ${ }^{13}$. Thus, to ease the low back pain from the patient's life and counteract limitations in daily activities, it is essential to understand the reason for low back pain and observe purposely the bodily movements that are causing pain and limitations ${ }^{15}$. Careful examinations by physical therapists, inside the light of imaging studies, can assist with the patients being diagnosed with a known and treatable reason for low back-pain ${ }^{16}$. As discussed above, one of the major reasons for back pain is altered spinal patterns of movement caused by changes in spinal structure, i.e. change in the curvatures of spine, caused by mostly muscular imbalances $^{17}$. Myofascial release (MFR) is among one of the most effective methods in manual medicine field in easing low back pain. MFR technique is widely used to manage pain associated with musculoskeletal lesions, hence the series of therapeutic effects are maximized by comforting muscles at the lesion as much as probable ${ }^{18}$. Another technique introduced by James Cyriax, termed as Instrument Assisted Soft Tissue Mobilization (IASTM), which is one of the latest treatment methods, is used for management of myofascial restriction $^{18}$, is applied using particularly designed, special instruments to provide mobilizing effects to soft tissue such as scar tissue, or myofascial adhesions in order to improve range of motion (ROM) and enhance pain tolerance ${ }^{19}$. Another form of treatment regime, being utilized is known as core stability exercises, which are used to treat muscular imbalances and weakness. Core stability exercises regimes fortify spinal musculature, by emphasizing on lumbo-pelvic region especially on the core muscles e.g., transverses abdominis, multifidus ${ }^{20}$. Evidence supports that core stability workouts, which are believed to target these critical muscles, are successful for the treatment and management of low back pain. It was also proved by evidence that core stability exercises are more reasonable than common workouts in treating low back pain within the general population. Patients with low back pain for more than 6 months duration have restrained trunk movements within the lumbosacral locale, which irritates the levels of lumbar region muscle in paraspinal muscles and multifidus ${ }^{14}$. These changes increase lumbar instability and increase the recurrence of low back pain. In this way, spinal 
extensors are significant in improving lumbar strength. Disintegrated physical functions in lumbo-sacral region may lead to weakened muscle strength inside the lumbar region, in this manner causing back pain ${ }^{20}$. On the preface of qualities, the muscles, that are responsible for spinal stability, can be separated into two groups. One of the group of muscles known as the local stabilizing muscles which are the deep core muscles, including the transverses abdominis, lumbar multifidus, and quadrates lumborum. These muscles are suitable for spinal stability and give precise motor control $^{20}$. Another group of muscles constitutes superficial core muscles, known as global stabilizing muscles, including the abdominal recti, internal oblique muscle and outer oblique muscles, erector spinae, quadrates lumborum, and muscles of hip region. High torque is produced by these muscles, to balance outside forces influencing the spine ${ }^{14}$. These muscles are responsible for to keep up with segmental strength, secure the spine, and decline weight affecting the lumbar vertebrae and intervertebral plates 20 .

\section{METHODOLOGY}

\section{Clinical Setting}

The study was conducted in the Department Of Rehabilitation Sciences, Dr. Ziauddin Hospital, Clifton, Karachi.

\section{Target Population}

Both male and female patients with Low back pain.

\section{Study Design \\ Randomized Control Trial (RCT).}

\section{Duration of Study}

The study was completed during a period of six month.

\section{Sampling Technique \\ Simple Random Sampling Technique.}

\section{Sample Size}

Sample size is calculated by using WHO software for samples size calculation. Taking previous study "Efficacy of progressive core strengthening exercise on functional endurance tests and hypertrophy of multifidus, transverses abdominis in healthy female subjects with low core endurance", using confidence interval of $95 \%$ and bound of error of $0.05 \%$, a total sample size of 44 is calculated ${ }^{21}$.

\section{Sample Selection}

The sample population was obtained using the following inclusion and exclusion criteria:

\section{Inclusion criteria}

- Both male and females between age, group of 18-60 years with low back pain referred to the department of rehabilitation sciences ${ }^{20}$.

\section{Exclusion criteria ${ }^{20}$}

- Post Spinal surgery/instrumentation.

- Medical "red flags" (e.g. Rheumatoid arthritis, osteoporosis, Fracture, Tumor) Prolonged steroid use and bone tissue infection or malignancy.

- Radiculopathy.

- Acute Traumatic injury of spine/lower extremity.

- Patient under anti-coagulant therapy.

- Any neurological or psychological deficit.

- Any Medico-legal Issues.

\section{Ethical Consideration}

All the data collected was kept confidential. The participants were informed about the risk and benefits of the study, and their right to quit at any time.

\section{RESULTS}

A total number of 44 participants with mean age of $45.34 \pm 5.2$ recruited comprised of $34 \%$ males and $66 \%$ females divided into Group $A(n=22)$ and $B$ $(n=22)$ respectively. The simple main effects analysis showed that the Core Stability Exercises applied with Myofascial release technique group proved to be more effective than the Core Stability Exercises group. The patients in the group B reported a mean difference (2.73 from 7.18 ) in their pain reduction and (19.09 from 37.91) mean reduction in functional disability and mean increase in muscle mass of Right Lumber Multifidus $(2.5 \mathrm{~cm}$ from $1.59 \mathrm{~cm})$ and Left Lumber Multifidus mean $(1.96 \mathrm{~cm}$ from $1.55 \mathrm{~cm})$ as shown in the NPRS (Fig-1), ODI (Fig-2) and Rehabilitative Ultrasound (Fig-3,4) scores in week 6, whereas patients in the group A reported a mean difference (4.05 from 7.55 ) in their pain reduction and (25.55 from 40.18) mean reduction in functional disability and mean increase in muscle mass of Right Lumber Multifidus $(2.2 \mathrm{~cm}$ from $1.68 \mathrm{~cm})$ and Left Lumber Multifidus mean $(2.02 \mathrm{~cm}$ from $1.64 \mathrm{~cm})$ in their NPRS (Fig-1), ODI (Fig-2) and Rehabilitative Ultrasound (Fig-3,4) scores in week 6.

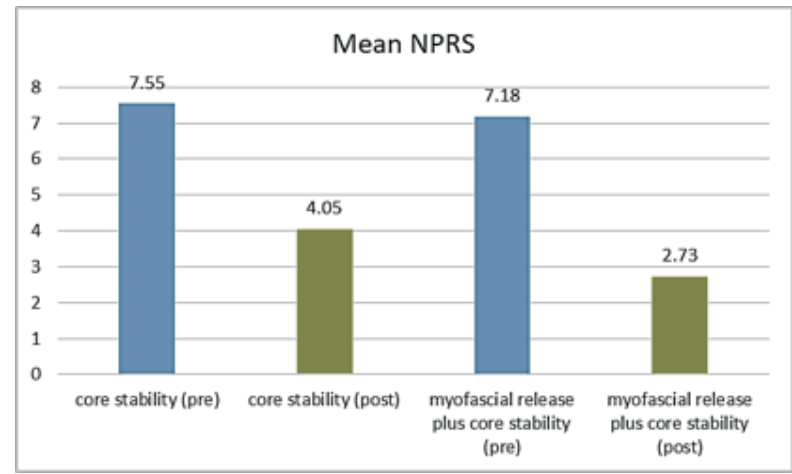

Figure-1 represents mean values of Numeric Pain Rating Scale of both groups, differentiating between Pre and Post intervention values.a 


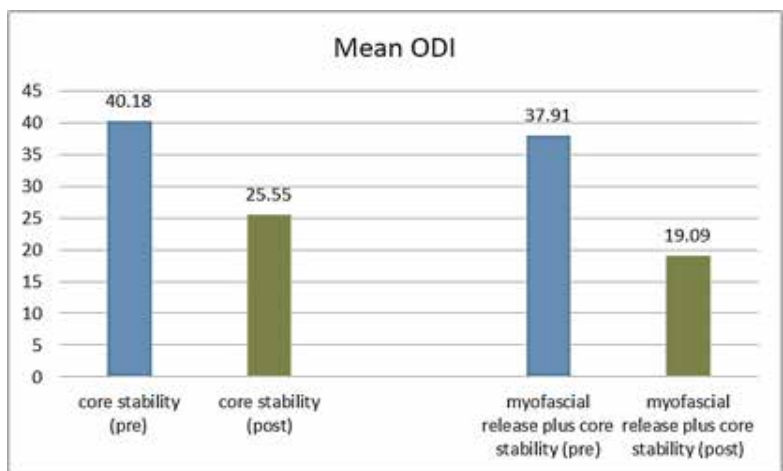

Figure-2 represents mean values of Oswestry disability index (ODI) of both groups, differentiating between Pre and Post intervention values.

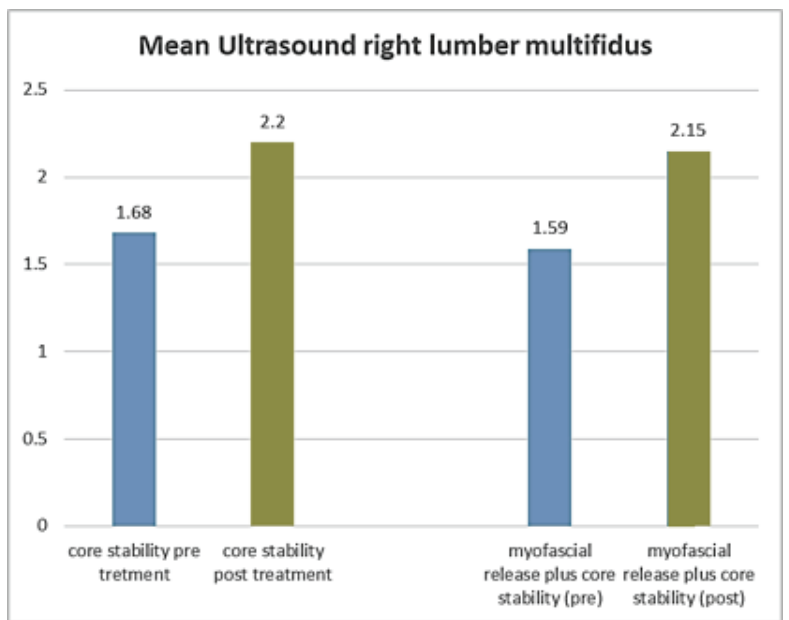

Figure-3 represents mean values of paired t-test Samples right lumber multifidus Ultrasound characteristics of both groups, differentiating between Pre and Post intervention values.

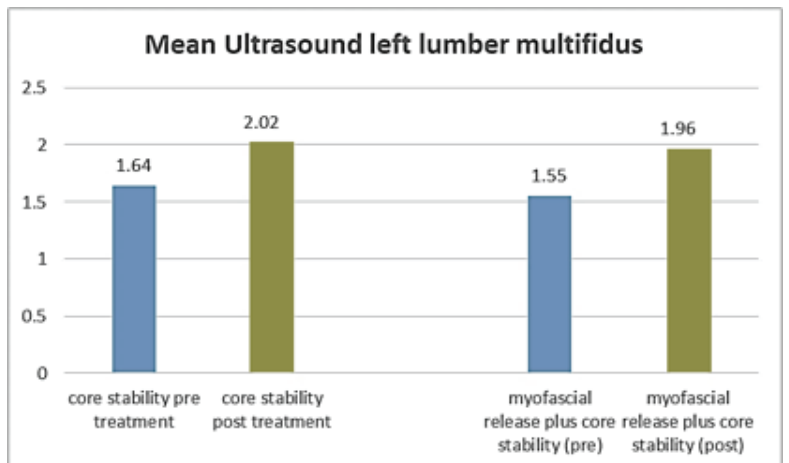

Figure-4 represents mean values of paired t-test Samples left lumber multifidus Ultrasound characteristics of both groups, differentiating between Pre and Post intervention values.

\section{DISCUSSION}

The study provided a mean to determine the efficacy of Core Stability Exercises and Myofascial
Release Technique for the management of Low back pain. The understanding of the Data analysis has revealed the ways and provided insights about the prescription of exercise interventions by physiotherapists for patients with low back pain. The thesis documented here has potential implications and significance to physical therapy education and clinical practice, as a basis of future research and discussion can be formed. Outcomes of this study revealed that statistically exercise intervention applied to both groups, evidenced to be effective in management of low back pain, but clinically, the evaluation revealed that there was greater pain reduction in Core Stability Exercises applied with Myofascial release technique group as compared to core stabilization exercise group. The study results revealed that core stability exercises reduced pain and augmented the daily functional activities in low back pain patients. Decrease in level of disability and pain in both groups was shown in the results of their study. The ability of the core muscles is enhanced by core stability exercises, therefore resulting in post-intervention decreased perception of pain and improved functional outcomes in subjects with low back pain. A moderate level decrease in pain perception was demonstrated by the subjects that were allocated to core stabilization group. The motor control exercises proved to be superior over electrotherapeutic modalities in the management of chronic non-specified low back pain, as established by Yang, $\mathrm{H}$. et al., in their study. The activation of the transversus abdominus and multifidus was targeted using Specific exercises performed on the Subjects in treatment group ${ }^{24}$. After the establishment of appropriate muscular control, more complex functional task aiming the activation of the core muscles were performed on the subjects in order to progress the with the treatment regime and target the activation of core muscles. Short wave diathermy and placebo ultrasound therapy for 10 minutes over 08 treatment session for 12 week was applied to the control group. Results showed considerable amount of pain reduction, measured on NPRS and disability measured on Roland-Morris Disability Questionnaire across the two groups. This reduction in values was clinically more significant in treatment group compared to control group, similar to our study ${ }^{13}$. The condition of the lumbar multifidus muscles can best be can best demonstrated by Magnetic Resonance Imaging, as suggested by Freeman et al.; However, ultrasound imaging is used, as it is already utilized and demonstrated in other studies ${ }^{12}$. According to our results, the lumbar multifidus Cross Sectional Area was greater in Stability Exercises applied with Myofascial release technique Group than Core Stability Exercises Group. Similar results in research were discussed by Areeudomwong, P., et al., indicating that dysfunction of Lumbar multifidus muscle is the result of spinal pain inhibition. In association with reduction in LBP, it is also essential to 
strengthen the multifidus muscles for the reason that they play important role in lumbar spine segmental stability and atrophy of lumbar multifidus muscles may contribute to the high recurrence rate of low back pain ${ }^{23}$. In our study, Cross sectional area of lumbar multifidus increased after 6 weeks of a specific core stabilization exercise program in both groups with low back pain. As mentioned in previous studies, patients with Low Back Pain had significantly smaller multifidus cross sectional area at the lowest two vertebral levels than asymptomatic subjects ${ }^{13}$. According to our study, specific retraining improved multifidus muscle Cross sectional area, accompanied with a decrease in pain. Classic trunk-strengthening exercises involve activation of the muscles. Such strengthening exercises have proved to be different from the stability exercises, which are intended to stabilize muscles, primarily by low-level isometric activation, followed by progressive integration into everyday activities. In our study, the myofascial release along with core stabilization group showed significantly greater improvement, when compared to the other group, as the ability to recruit the lumbar multifidus, though the difference was largely accounted for by a worsening in the other groups. Efficient increase in strength of multifidus muscle and reduced level of pain in patients with low back pain was associated with the performance of core stabilization exercise program, as confirmed by this study. It was also emphasized that the evaluation of disability scale levels are primarily based on functional activities that are a daily concern of LBP patients ${ }^{25}$. Thus, even though the core training and trunk balance exercises are challenging activities for individuals, they can reduce the level of disability. Moreover, patients more easily perceive the effectiveness of such core training and trunk balance exercises, than that of other pain reduction methods. No difference in the VAS pain scores between experimental and control groups was reported by Kawi, J., et al.; however, there may have been undetectable substantial improvement because of the low pretest scores of both groups. In spite of the reduction in the level of pain is indicative of that the functional disability of the CLBP patients had improved evidently ${ }^{25}$. Hence, our findings are In accordance with the findings of other studies. The strength of our study was the use of Rehabilitative ultrasound for the measurement of Muscle cross sectional area and thickness of spinal muscles. The limitation of the study included a small sample size. Moreover, the results could have been obtained after one month duration from application of intervention, in order to understand the long-term effects of intervention. Biasness based on gender was faced because of random sampling, which effect on the results has based on the differences between Muscle cross sectional area in males and females. The factor of patient biasness could also not be controlled.

\section{CONCLUSION}

The study focused on multiple modes of exercise programs focused on core stability. The present study has proven to provide significant improvement in low back pain, spinal muscle thickness and functional disability in patients with low back pain when treated with core stability exercise with Myofascial Release technique as compared to core stability exercise alone.

\section{REFERENCES}

[1] Hurwitz EL, Randhawa K, Yu H, Côté P, Haldeman S. The Global Spine Care Initiative: a summary of the global burden of low back and neck pain studies.

Eur Spine J 2018;27(6):796-801.

[2] Overaas CK, Johansson MS, de Campos TF, Ferreira ML, Natvig B, Mork PJ, Hartvigsen J. Prevalence and pattern of co-occurring musculoskeletal pain and its association with back-related disability among people with persistent low back pain: protocol for a systematic review and meta-analysis. Systematic reviews. 2017;6(1):258.

[3] Aboagye E, Hagberg J, Axén I, Kwak L, Lohela-Karlsson M, Skillgate E, Dahlgren G, Jensen I. Individual preferences for physical exercise as secondary prevention for non-specific low back pain: A discrete choice experiment. Plos one. 2017;12(12):e0187709.

[4] Deckers K, De Smedt K, van Buyten JP, Smet I, Eldabe S, Gulve A, Baranidharan G, de Andrès J, Gilligan C, Jaax K, Heemels JP. Chronic low back pain: restoration of dynamic stability. Neuromodulation: Technology at the Neural Interface. 2015;18(6):478-86.

[5] Yiengprugsawan V, Hoy D, Buchbinder R, Bain C, Seubsman SA, Sleigh AC. Low back pain and limitations of daily living in Asia: longitudinal findings in the Thai cohort study. BMC musculoskeletal disorders. 2017;18(1):19.

[6] Kashif M, Ain QU, Heera SA. Prevalence of LBP in male factory workers of Nishat Mills limited, Sheikhupura. Pakistan. J Pharm Altern Med. 2014;3:13-6.

[7] Sailaja AK. Treatment for low back pain attributed to underlying presumptive etiology. Am J Drug Deliv Therap. 2015;2(1):001-8.

[8] Casser HR, Seddigh S, Rauschmann M. Acute Lumbar Back Pain: Investigation, Differential Diagnosis, and Treatment. Deutsches Ärzteblatt International. 2016;113(13):223.

[9] Chenot JF, Greitemann B, Kladny B, Petzke F, Pfingsten M, Schorr SG. Non-specific low back pain. Deutsches Ärzteblatt International. 2017;114(51-52):883.

[10] Chang WD, Lin HY, Lai PT. Core strength training for patients with chronic low back pain. J Phys Ther Sci. 2015;27(3):619-22. 
[11] Searle A, Spink M, Ho A, Chuter V. Exercise interventions for the treatment of chronic low back pain: a systematic review and meta-analysis of randomised controlled trials. Clinical rehabilitation. 2015 29(12):1155-67.

[12] Worsley PR, Kitsell F, Samuel D, Stokes M. Validity of measuring distal vastus medialis muscle using rehabilitative ultrasound imaging versus magnetic resonance imaging. Manual therapy. 2014;19(3):259-63.

[13] Stuber KJ, Bruno P, Sajko S, Hayden JA. Core stability exercises for low back pain in athletes: a systematic review of the literature. Clin J Sport Med. 2014;24(6):448-56.

[14] Potier T, Tims E, Kilbride C, Rantell K. Evaluation of an evidence based quality improvement innovation for patients with musculoskeletal low back pain in an accident and emergency setting. BMJ Open Quality. 2015;4(1):U205903w2411.

[15] Gordon R, Bloxham S. A systematic review of the effects of exercise and physical activity on non-specific chronic low back pain. InHealthcare 2016 (Vol. 4, No. 2, p. 22). Multidisciplinary Digital Publishing Institute.

[16] Shah SG, Kage V. Effect of seven sessions of posterior-to-anterior spinal mobilisation versus prone press-ups in non-specific low back pain-randomized clinical trial. J Clin Diagn Res: JCDR. 2016;10(3):YC10.

[17] Gasibat Q, Simbak N, Aziz AA, Musa RM. A comparative efficacy between strength and Stabilisation training programmes in the Improvement of trunk and hip muscles Activations of healthy females subject: An implication for the rehabilitation Practitioners. Int J Life Sci Pharma Res. 2017 7(4):L30-8.

[18] Ajimsha MS, Al-Mudahka NR, Al-Madzhar JA. Effectiveness of myofascial release: Systematic review of randomized controlled trials. J

[19] Bodyw Mov Ther. 2015;19(1):102-12.

Baker RT, Nasypany A, Seegmiller JG, Baker JG. Instrument-assisted soft tissue mobilization treatment for tissue extensibility dysfunction. Int

[20] J Athl Ther Train. 2013;18(5):16-21. Wang $X Q$, Zheng JJ, YU ZW, Bi X, Lou SJ, LiU J, Cai B, Hua YH, Wu M, Wei ML, Shen HM. A meta-analysis of core stability exercise versus general exercise for chronic low back pain.

[21] Plos one. 2012;7(12):e52082.

Kulandaivelan S, Chaturvedi R, Moolchandani $\mathrm{H}$. Efficacy of progressive core strengthening exercise on functional endurance tests and hypertrophy of multifidus, transverses abdominis in healthy female subjects with low core endurance. JESP. 2014;10(2):114.

[22] Akhtar MW, Karimi H, Gilani SA. Effectiveness of core stabilization exercises and routine exercise therapy in management of pain in chronic non-specific low back pain: A randomized controlled clinical trial. Pak J Med Sci. 2017;33(4):1002.

[23] Areeudomwong P, Puntumetakul R, Jirarattanaphochai K, Wanpen S, Kanpittaya J, Chatchawan U, Yamauchi J. Core stabilization exercise improves pain intensity, functional disability and trunk muscle activity of patients with clinical lumbar instability: a pilot randomized controlled study. J Phys Ther Sci. 2012;24(10):1007-12.

[24] H, Haldeman S. Behavior-related factors associated with low back pain in the US adult population. Spine. 2018;43(1):28-34.

[25] Kawi J. Self-management and self-management support on functional ablement in chronic low back pain. Pain Management Nursing. 2014;15(1):41-50. 